\title{
A PARTITION THEOREM FOR PAIRS OF FINITE SETS
}

\author{
THOMAS JECH AND SAHARON SHELAH
}

\section{INTRODUCTION}

A branch of combinatorics called Ramsey theory deals with phenomena of the following kind: If a sufficiently large set of objects is endowed with a certain structure then a large subset can be found whose all elements are "alike."

A simple instance is the pigeon-hole principle: If there are more pigeons than pigeon-holes then some pigeon-hole is occupied by more than one pigeon. Another is this (the finite Ramsey theorem): For every integer $k>2$ there is an integer $n$ with the property that if $A$ is a set of at least $n$ elements and if the set of all (unordered) pairs $\{a, b\} \subset A$ is divided into two classes then there is a subset $H \subset A$ of size $k$ such that all pairs $\{a, b\} \subset H$ belong to the same class.

Many such principles have been formulated and proved, with applications in various branches of mathematics; most are variants of Ramsey's Theorem [2].

Ramsey's Theorem states (in particular) that every partition of the set $[\mathbf{N}]^{2}$ (into finitely many pieces) has an infinite homogeneous set, i.e., a set $H \subseteq \mathbf{N}$ cofinal in $(\mathbf{N},<)$ such that $[H]^{2}$ is included in one piece of the partition. The following generalization of Ramsey's Theorem was suggested in [3]:

Let $A$ be an infinite set, and let $[A]^{<\omega}$ denote the set of all finite subsets of $A$. A set $H \subseteq[A]^{<\omega}$ is cofinal in $[A]^{<\omega}$ if for every $x \in[A]^{<\omega}$ there exists a $y \in H$ such that $x \subseteq y$. Note that if a cofinal set $H$ is partitioned into two pieces, $H=H_{1} \cup H_{2}$, then at least one of the two sets $H_{1}, H_{2}$ is cofinal.

Let $F:\left[[A]^{<\omega}\right]^{2} \rightarrow\{1, \ldots, k\}$ be a partition of pairs of finite subsets of $A$; a set $H \subseteq[A]^{<\omega}$ is homogeneous for $F$ if all pairs $(a, b) \in[H]^{2}$ with the property that $a \subset b$ belong to the same piece of the partition, i.e.,

$$
F\left(x_{1}, x_{2}\right)=F\left(y_{1}, y_{2}\right)
$$

whenever $x_{1}, x_{2}, y_{1}, y_{2} \in H$ and $x_{1} \subset x_{2}, y_{1} \subset y_{2}$.

The question raised in [3] asked whether for every infinite $A$, every partition of $\left[[A]^{<\omega}\right]^{2}$ has a cofinal homogeneous set.

Received by the editors February 1, 1990 and, in revised form, October 9, 1990.

1991 Mathematics Subject Classification. Primary 03E05, 04A20, 05 C55.

The first author was supported by NSF Grant DMS-8614447 and by BSF.

The second author was supported at MSRI by NSF Grant 8505550 and by BSF. Publ. No. 392 . 
It is not difficult to see that if $A$ is countable, then $[A]^{<\omega}$ has a cofinal subset of order type $\omega$ and so $[A]^{<\omega}$ satisfies the partition property as a consequence of Ramsey's Theorem. For an arbitrary $A$, the partition property in question is a generalization of Ramsey's Theorem for pairs.

We answer the question in the affirmative in the case when $|A|=\aleph_{1}$.

Theorem 1. If $|A|=\aleph_{1}$ then every partition of $\left[[A]^{<\omega}\right]^{2}$ into finitely many pieces has a cofinal homogeneous set.

The question remains open for sets of size greater than $\aleph_{1}$. By an unpublished theorem of Galvin, Martin's Axiom implies the partition property for all sets $A$ of cardinality less than $2^{\aleph_{0}}$.

More generally, let $S$ be a partially ordered set, and assume that $S$ is directed and does not have a maximal element. A set $H \subseteq S$ is cofinal in $S$ if for every $x \in S$ there exists a $y \in H$ such that $x \leq y$. Let $r \geq 2$ and $k \geq 2$, and let $F:[S]^{r} \rightarrow\{1, \ldots, k\}$ be a partition of $r$-tuples in $S$. A set $H \subseteq S$ is homogeneous for $F$ if for all $x_{1}, \ldots, x_{r}$ and $y_{1}, \ldots, y_{r}$ such that $x_{1}<\cdots<$ $x_{r}$ and $y_{1}<\cdots<y_{r}$ we have

$$
F\left(x_{1}, \ldots, x_{r}\right)=F\left(y_{1}, \ldots, y_{r}\right) .
$$

Using the standard arrow notation, the formula

$$
S \rightarrow(\text { cofinal subset })_{k}^{r}
$$

states that for every partition $F:[S]^{r} \rightarrow\{1, \ldots, k\}$ there exists a cofinal subset $H$ of $S$ homogeneous for $\mathrm{F}$.

The following is an unpublished result of Galvin [1]:

Theorem 2 (F. Galvin). Assume $M A(\kappa)$. Let $S$ be a partially ordered set of power $\kappa$, which is directed, and suppose for all $a \in S,\{b \in S: b<a\}$ is finite. Let $f:\{(x, y) \in S \times S: x<y\} \rightarrow$ red, blue $\}$. Then there is a cofinal $H \subseteq S$ such that $f$ is constant on $\{(x, y) \in H \times H: x<y\}$.

Galvin's method admits a generalization to partitions of $r$-tuples, for any $r \geq 2$ (see the proof of Theorem 4 below). Thus assuming Martin's Axiom the following holds

Theorem $2^{\prime}$. Let $S$ be a directed partially ordered set of cardinality less than $2^{\aleph_{0}}$, without maximal element and such that for every $a \in S$ the set $\{x \in S$ : $x<a\}$ is finite. Then

$$
S \rightarrow(\text { cofinal subset })_{k}^{r} \text { for all } r, k<\omega .
$$

Note that every partially ordered set $S$ with the properties stated above is isomorphic to a cofinal subset of $[S]^{<\omega}$.

The statement that for every cofinal $S \subseteq\left[\omega_{1}\right]^{<\omega}, S \rightarrow(\text { cofinal subset })_{2}^{2}$ is not a theorem of ZFC, as by an unpublished result of Laver [4] a counterexample exists under the assumption of the continuum hypothesis: 
Theorem 3 (R. Laver). Let $\kappa$ be a cardinal such that $\kappa^{\aleph_{0}}=\kappa$. Then there exist a cofinal set $S \subset[\kappa]^{<\omega}$ and a partition $F:[S]^{2} \rightarrow\{1,2\}$ such that no cofinal subset of $S$ is homogeneous for $F$.

With Laver's permission we include the proof of Theorem 3 below.

We say that a partially ordered set $S$ has finite character if $S$ has a cofinal set $S^{\prime}$ such that every $x \in S^{\prime}$ has only finitely many predecessors in $S^{\prime}$. Thus Galvin's theorem implies that $S \rightarrow$ (cofinal subset $)_{k}^{r}$ holds for every set $S$ of size $\aleph_{1}$ that has finite character if Martin's Axiom holds together with $2^{\aleph_{0}}>\aleph_{1}$, and Laver's theorem implies that if $2^{\aleph_{0}}=\aleph_{1}$ then a partial order $S$ exists that has size $\aleph_{1}$ and finite character but $S \rightarrow(\text { cofinal subset })_{2}^{2}$ fails.

Theorem 4. In the Cohen model for $2^{\aleph_{0}}=\aleph_{2}$ the following statements are equivalent for every directed set of cardinality $\aleph_{1}$ :

(1) $S \rightarrow(\text { cofinal subset })_{2}^{2}$,

(2) $S \rightarrow(\text { cofinal subset })_{k}^{r}$ for all $r, k<\omega$,

(3) $S$ has finite character.

The consistency proof of “(3) implies (2)" is essentially Galvin's result; we will show that (1) implies (3) in the Cohen model.

\section{Proof of Theorem 1}

Throughout this section we consider a fixed partition $F:\left[\left[\omega_{1}\right]^{<\omega}\right]^{2} \rightarrow$ $\{1, \ldots, k\}$. The pairs $\{x, y\}$ such that $x \subset y$ are divided into two classes; we shall refer to these two classes as colors.

We reserve lower case letters such as $a, b, c$ for finite subsets of $\omega_{1}$ and capital letters such as $A, B, C$ for at most countable subsets of $\omega_{1}$.

A partial coloring of a finite set $a$ is a function $f$ whose domain is a set of subsets of $a$ and whose values are in the set $\{1, \ldots, k\}$. A total coloring of $a$ is a partial coloring whose domain is the set of all subsets of $a$.

If $a \subset b$ and if $f$ is a partial coloring of $a$ then $b$ is $f$-correct if for every $x \in \operatorname{dom}(f)$, the pair $(x, b)$ has the color $f(x)$ (i.e., $F(x, b)=f(x))$.

If $a \subseteq A$ then $b$ is an A-extension of $a, a \leq_{A} b$, if $a \subseteq b$ and $b \cap A=a$. An $A$-extension $b$ of $a$ is proper if $a \subset b$.

We shall consider pairs $(a, A)$ where $a$ is finite, $A$ is at most countable and $a \subseteq A$. If $a \subseteq A$ and $b \subseteq B$ then

$$
(a, A) \leq(b, B)
$$

means that $A \subseteq B$ and $b$ is an $A$-extension of $a$. Note that $\leq$ is transitive.

Definition 2.1. Let $a \subseteq A$, and let $f$ be a partial coloring of $a$. We say that the pair $(a, A)$ is good for $f$ if for every $(b, B) \geq(a, A)$ there exists a proper $B$-extension $c$ of $b$ that is $f$-correct.

Remark. If $(a, A)$ is good for $f$ and if $f^{\prime} \subseteq f$ and $\left(a^{\prime}, A^{\prime}\right) \geq(a, A)$ then $\left(a^{\prime}, A^{\prime}\right)$ is good for $f^{\prime}$. 
Lemma 2.2. For every $(b, B)$ there exist a total coloring $g$ of $b$ and some $(c, C) \geq(b, B)$ such that $(c, C)$ is good for $g$.

Moreover, we may require that $c$ is a proper $B$-extension of $b$ and is $g$ correct.

Proof. First assume that $g$ and $(c, C) \geq(b, B)$ are as claimed in the first part of the lemma. Then there is some $d>_{C} c$ that is $g$-correct, and $(d, C \cup d)$ is good for $g$. Hence it suffices to find for each $(b, B)$ a total coloring $g$ of $b$ and some $(c, C) \geq(b, B)$ good for $g$.

Thus assume that the lemma fails and let $(b, B)$ be such that for every total coloring $g$ of $b$, no $(c, C) \geq(b, B)$ is good for $g$.

There are finitely many total colorings $g_{1}, \ldots, g_{m}$ of $b$. We construct a sequence $\left(b_{i}, B_{i}\right), i=1, \ldots, m$, so that

$$
(b, B) \leq\left(b_{1}, B_{1}\right) \leq \cdots \leq\left(b_{m}, B_{m}\right)
$$

as follows:

As $(b, B)$ is not good for $g_{1}$, there exists some $\left(b_{1}, B_{1}\right) \geq(b, B)$ such that no proper $B_{1}$-extension of $b_{1}$ is $g_{1}$-correct.

Next, as $\left(b_{1}, B_{1}\right)$ is not good for $g_{2}$, there exists some $\left(b_{2}, B_{2}\right) \geq\left(b_{1}, B_{1}\right)$ such that no proper $B_{2}$-extension of $b_{2}$ is $g_{2}$-correct.

And so on. For each $i=1, \ldots, m$, no proper $B_{i}$-extension of $b_{i}$ is $g_{i}$ correct.

Now let $c$ be an arbitrary proper $B_{m}$-extension of $b_{m}$. Let us consider the following total coloring $g$ of $b$ :

$$
g(x)=F(x, c) \quad(\text { the color of }(x, c)) .
$$

We have $g=g_{i}$ for some $i \leq m$. It is now clear that $c$ is a $g_{i}$-correct proper $B_{i}$-extension of $b_{i}$, a contradiction.

Lemma 2.3. If $(a, A)$ is good for $f$, then for every $(b, B) \geq(a, A)$ there exists a total coloring $g$ of $b$ extending $f$ and some $(c, C) \geq(b, B)$ such that $c$ is $a$-correct proper B-extension of $b$ and $(c, C)$ is good for $g$.

Proof. The proof proceeds as in Lemma 2.2, the difference being that we consider only the total colorings $g_{1}, \ldots, g_{m}$ of $b$ that extend $f$. After having constructed $\left(b_{1}, B_{1}\right) \leq \cdots \leq\left(b_{m}, B_{m}\right)$, we find (because $(a, A)$ is good for $f$ and $\left.(a, A) \leq\left(b_{m}, B_{m}\right)\right)$ a proper $B_{m}$-extension $c$ of $b_{m}$ that is $f$-correct. Then $g$ (defined as above) extends $f$ and so $g=g_{i}$ for some $i \leq m$. The rest of the proof is as before.

We shall use Lemmas 2.2 and 2.3 to construct an end-homogeneous cofinal set $H \subseteq\left[\omega_{1}\right]^{<\omega}$.

Definition 2.4. A set $H$ is end-homogeneous if for all $x, y, z \in H$, if $x \subset y$ and $x \subset z$ then $(x, y)$ and $(x, z)$ have the same color.

Note that if $H$ is a cofinal end-homogeneous set, then one of the sets $H_{i}=\{a \in H: F(a, x)=i$ for all $x \in H$ such that $a \subset x\} \quad(i=1, \ldots, k)$ 
is cofinal and homogeneous. It follows that it suffices to construct a cofinal end-homogeneous set.

Definition 2.5. An approximation is a triple $(A, G, H)$ where $A$ is an infinite countable subset of $\omega_{1}, G$ and $H$ are disjoint cofinal subsets of $[A]^{<\omega}, H$ is end-homogeneous, and for every $a \in G,(a, A)$ is good for $f_{a}^{H}$ where $f_{a}^{H}$ is the partial coloring of $a$ defined on $\{x \subset a: x \in H\}$ by

$$
f_{a}^{H}(x)=\text { the color of }(x, y) \text {, where } y \text { is any } y \in H \text { such that } x \subset y \text {. }
$$

Let

$$
(A, G, H) \leq\left(A^{\prime}, G^{\prime}, H^{\prime}\right)
$$

mean that $A \subseteq A^{\prime}, G \subseteq G^{\prime}$, and $H \subseteq H^{\prime}$. We want to construct an increasing sequence of approximations $\left(A_{\alpha}, G_{\alpha}, H_{\alpha}\right)$ such that $\bigcup_{\alpha} A_{\alpha}=\omega_{1}$. Then $H=$ $\bigcup_{\alpha} H_{\alpha}$ is an end-homogeneous set, cofinal in $\left[\omega_{1}\right]^{<\omega}$.

It is easy to verify that if $\lambda$ is a countable limit ordinal, and if $\left(A_{\alpha}, G_{\alpha}, H_{\alpha}\right)$, $\alpha<\lambda$, is an increasing sequence of approximations, then $\left(\cup_{\alpha} A_{\alpha}, \cup_{\alpha} G_{\alpha}\right.$, $\left.\bigcup_{\alpha} H_{\alpha}\right)$ is an approximation. Thus to complete the proof, it suffices to prove the following two lemmas:

Lemma 2.6. There exists an approximation.

Lemma 2.7. Let $(A, G, H)$ be an approximation and let $\xi \in \omega_{1}-A$ be arbitrary. Then there is an approximation $(\bar{A}, \bar{G}, \bar{H}) \geq(A, G, H)$ such that $\xi \in \bar{A}$.

Proof of Lemma 2.6. We construct $A$ as the union of a sequence $c_{0} \subset c_{1} \subset$ $\cdots \subset c_{n} \subset \cdots$ of finite sets as follows. Let $b_{0}$ be an arbitrary finite subset of $\omega_{1}$. By Lemma 2.2, there exist a total coloring $g_{0}$ of $b_{0}$ and some $\left(c_{0}, C_{0}\right)$ such that $\left(c_{0}, C_{0}\right)$ is good for $g_{0}$ and $c_{0} \supset b_{0}$ is $g_{0}$-correct.

Now let $n \geq 0$; we have constructed $\left(c_{0}, C_{0}\right), \ldots,\left(c_{n}, C_{n}\right)$ such that $c_{0} \subset$ $\cdots \subset c_{n}$. Fix for each $i \leq n$ an enumeration of $C_{i}$ of order-type $\omega$. Let $b_{n+1} \supseteq c_{n}$ be a finite set such that for each $i \leq n, b_{n+1}$ contains the first $n$ elements of $C_{i}$. This will guarantee that $\bigcup_{n=0}^{\infty} c_{n}=\bigcup_{n=0}^{\infty} C_{n}$.

By Lemma 2.2, there exist a total coloring $g_{n+1}$ of $b_{n+1}$ and some $\left(c_{n+1}\right.$, $\left.C_{n+1}\right)$ such that $\left(c_{n+1}, C_{n+1}\right)$ is good for $g_{n+1}$ and $c_{n+1} \supset b_{n+1}$ is $g_{n+1}$-correct.

We let $A=\bigcup_{n=0}^{\infty} C_{n}$. To construct $G$ and $H$, consider the partition $F$ restricted to the set $\left[\left\{c_{n}\right\}_{n=0}^{\infty}\right]^{2}$. By Ramsey's Theorem, $\left\{c_{n}\right\}_{n=0}^{\infty}$ has an infinite homogeneous (say, green) subsequence. Let us denote this subsequence

$$
d_{0} \subset e_{0} \subset d_{1} \subset e_{1} \subset \cdots \subset d_{i} \subset e_{i} \subset \cdots
$$

and let $G=\left\{d_{i}\right\}_{i=0}^{\infty}, H=\left\{e_{i}\right\}_{i=0}^{\infty}$. Clearly, $G$ and $H$ are disjoint cofinal subsets of $[A]^{<\omega}$. Moreover, $H$ is homogeneous, and we claim that for every $a \in G,(a, A)$ is good for $f_{a}^{H}$. Since $a=c_{n}$ for some $n,\left(c_{n}, C_{n}\right)$ is good for $g_{n}$ and $\left(c_{n}, C_{n}\right) \leq\left(c_{n}, A\right)$, it suffices to show that $f_{a}^{H} \subseteq g_{n}$. If $x \in \operatorname{dom} f_{a}^{H}$ then because $g_{n}$ is a total coloring of $b_{n}$ and $x=c_{m}$ for some $m<n$, and 
because $c_{n}$ is $g_{n}$-correct, we have $g_{n}(x)=$ the color of $(x, a)$, which is green, because both $x$ and $a$ are in the homogeneous sequence. But $f_{a}^{H}(x)$ is also green. Hence $f_{a}^{H} \subseteq g_{n}$, and $(A, G, H)$ is an approximation.

Proof of Lemma 2.7. We construct $\bar{A}$ as the union of a sequence $c_{0} \subset c_{1} \subset$ $\cdots \subset c_{n} \subset \cdots$ of finite sets as follows. First, we choose an increasing cofinal sequence $a_{0} \subset a_{1} \subset \cdots \subset a_{n} \subset \cdots$ in $G$. Let $b_{0}$ be some $A$-extension of $a_{0}$ such that $\xi \in b_{0}$. As $\left(a_{0}, A\right)$ is good for $f_{a_{0}}^{H}$, there exist a total coloring $g_{0}$ of $b_{0}$ extending $f_{a_{0}}^{H}$, an $A$-extension $c_{0}$ of $a_{0}$ such that $c_{0} \supset b_{0}$ and some $C_{0} \supseteq A \cup c_{0}$ such that $c_{0}$ is $g_{0}$-correct and $\left(c_{0}, C_{0}\right)$ is good for $g_{0}$.

Now assume that $\left(c_{n}, C_{n}\right)$ has been constructed and $c_{n}$ is an $A$-extension of $a_{n}$. Let $b_{n+1}$ be some $A$-extension of $a_{n+1}$ such that $b_{n+1} \supseteq c_{n}$. Moreover, we choose $b_{n+1}$ large enough to contain the first $n$ elements of each $C_{i}-A$, $i=0, \ldots, n$ (in some fixed enumeration). This will guarantee that $\bigcup_{n=0}^{\infty} c_{n}=$ $\bigcup_{n=0}^{\infty} C_{n}$.

As $\left(a_{n+1}, A\right)$ is good for $f_{a_{n+1}}^{H}$, there exist a total coloring $g_{n+1}$ of $b_{n+1}$ extending $f_{a_{n+1}}^{H}$, an $A$-extension $c_{n+1}$ of $a_{n+1}$ such that $c_{n+1} \supset b_{n+1}$ and some $C_{n+1} \supseteq A \cup c_{n+1}$ such that $c_{n+1}$ is $g_{n+1}$-correct and $\left(c_{n+1}, C_{n+1}\right)$ is good for $g_{n+1}$.

We let $\bar{A}=\bigcup_{n=0}^{\infty} C_{n}$. To construct $\bar{G}$ and $\bar{H}$, consider the partition restricted to the set $\left[\left\{c_{n}\right\}_{n=0}^{\infty}\right]^{2}$. By Ramsey's Theorem, $\left\{c_{n}\right\}_{n=0}^{\infty}$ has an infinite homogeneous (let us say green) subsequence. Let us denote this subsequence

$$
d_{0} \subset e_{0} \subset d_{1} \subset e_{1} \subset \cdots \subset d_{i} \subset e_{i} \subset \cdots
$$

and let $\bar{G}=G \cup\left\{d_{i}\right\}_{i=0}^{\infty}, \bar{H}=H \cup\left\{e_{i}\right\}_{i=0}^{\infty}$. Clearly, $\bar{G}$ and $\bar{H}$ are disjoint cofinal subsets of $[\bar{A}]^{<\omega}$, and $G=\bar{G} \cap[A]^{<\omega}$ and $H=\bar{H} \cap[A]^{<\omega}$. It remains to show that $\bar{H}$ is end-homogeneous, and that for every $a \in \bar{G},(a, \bar{A})$ is good for $f_{a}^{H}$.

To prove that $\bar{H}$ is end-homogeneous, we have to show that the color of $(x, y)$ for $x, y \in \bar{H}$ does not depend on $y$. If $x \in \bar{H}-H$, say $x=e_{i}$, then every $y \supset x$ in $\bar{H}$ is some $e_{m}$ and $\left(e_{i}, e_{m}\right)$ is green. If $x \in H$, then the color of $(x, y)$ is determined by $H$ and should be equal to $f_{a}^{H}(x)$ for any $a \supset x$ in $G$. We have to show that $\left(x, e_{i}\right)$ has this color for all $e_{i} \supset x$. So let $i$ be such that $e_{i} \supset x$; we have $e_{i}=c_{n}$ for some $n$. As $c_{n}$ is an $A$-extension of $a_{n}$, it follows that $x \subset a_{n}$. Since $c_{n}$ is $g_{n}$-correct and $g_{n} \supseteq f_{a_{n}}^{H}, c_{n}$ is $f_{a_{n}}^{H}$-correct. Therefore $\left(x, c_{n}\right)$ has color $f_{a_{n}}^{H}(x)$.

Finally, we prove that for every $a \in \bar{G},(a, \bar{A})$ is good for $f_{a}^{\bar{H}}$. If $a \in G$ then $f_{a}^{\bar{H}}$ is just $f_{a}^{H}$ because $\{x \subset a: x \in \bar{H}\}=\{x \subset a: x \in H\}$. Because $(a, A)$ is good for $f_{a}^{H}$ and $A \subseteq \bar{A},(a, \bar{A})$ is good for $f_{a}^{\bar{H}}$. So let $a \in \bar{G}-G$, say $a=d_{i}=c_{n}$. We know that $\left(c_{n}, C_{n}\right)$ is good for $g_{n}$ and $C_{n} \subseteq \bar{A}$, so it suffices to show that $f_{a}^{\bar{H}} \subseteq g_{n}$, and then it follows that $(a, A)$ is good for $f_{a}^{\bar{H}}$. 
So let $x \subset a$ be an element of $\bar{H}$. If $x \in H$ then, because $a=c_{n}$ is an $A$-extension of $a_{n}, x \subset a_{n}$ and so $x \in \operatorname{dom}\left(f_{a_{n}}^{H}\right)$. We already know that $\bar{H}$ is end-homogeneous, so $f_{a}^{H}(x)=f_{a_{n}}^{H}(x)=$ the color of $(x, y)$ for any $y \supset x$ in $\bar{H}$. Because $g_{n}$ is an extension of $f_{a_{n}}^{H}$, we have $f_{a}^{\bar{H}}(x)=g_{n}(x)$.

If $x \in \bar{H}-H$ then $x=c_{m}$ for some $m<n$ and $f_{a}^{H}(x)=$ green (because $x=e_{i}$ for some $i$ ). Now $g_{n}$ is a total coloring of $b_{n}$, and $b_{n} \supseteq c_{m}$, so $x \in \operatorname{dom}\left(g_{n}\right)$ and it remains to show that $g_{n}(x)=$ green. But $c_{n}$ is $g_{n}$-correct, and so $g_{n}(x)=$ the color of $\left(c_{m}, c_{n}\right)=$ green.

\section{Proof of Theorem 4}

We shall prove that the equivalence between the partition property and finite character, for directed partial orders of size $\aleph_{1}$, holds in the model $V[G]$ obtained by adding $\aleph_{2}$ Cohen reals to a ground model for ZFC.

We shall first prove that (3) implies (1) in the Cohen model, and then outline how the proof can be modified to show that (3) implies (2). Assume that $S$ is a directed partially ordered set of size $\aleph_{1}$ in the model $V[G]$, and assume that each $a \in S$ has finitely many predecessors. Let $F$ be a partition of $[S]^{2}$. As $|S|=|F|=\aleph_{1}, \quad V[G]$ is a generic extension of $V[S, F]$ by the Cohen forcing, and we may assume that $S$ and $F$ are in the ground model. Thus it suffices to prove that adding $\aleph_{1}$ Cohen reals produces a cofinal homogeneous set for $F$.

In fact, we define a forcing notion $P$ that produces a generic cofinal homogeneous set for $F$, and then show that $P$ is equivalent to adding $\aleph_{1}$ Cohen reals. The forcing notion $P$ is essentially the one used by Galvin in his proof of the partition property for $\left[\omega_{1}\right]^{<\omega}$ from Martin's Axiom.

Let $D$ be an ultrafilter on $S$ with the property that for every $a \in S,\{x \in$ $S: a \leq x\} \in D$. We say that $a \in S$ is $r e d$, if for $D$-almost all $x>a,(a, x)$ is red; otherwise $a$ is green. Either almost all $a \in S$ are red, or almost all are green; let us assume that almost all $a \in S$ are red. A forcing condition in $P$ is a finite red-homogeneous set of red points. A condition $p$ is stronger than $q$ if $p \supseteq q$ and if for no $x \in p-q$ and no $y \in q$ we have $x<y$.

Using the ultrafilter $D$ one can easily verify that for every $p \in P$ and every $a \in S$ there exists some $x \geq a$ such that $p \cup\{x\}$ is a condition stronger than $p$. Therefore a generic set is a cofinal homogeneous set.

We shall finish the proof by showing that the forcing $P$ is equivalent to adding $\aleph_{1}$ Cohen reals. Let $S_{\alpha}, \alpha<\omega_{1}$, be an elementary chain of countable submodels of $(S,<$, red $)$, with limit $S$. For each $\alpha$, let $P_{\alpha}=\{p \in P: p \subset$ $\left.S_{\alpha}\right\}$. Each $P_{\alpha}$ is a countable forcing notion, therefore equivalent to adding a Cohen real. It suffices to prove that every maximal antichain in $P_{\alpha}$ is a maximal antichain in $P$. This will follow from this claim: For every $p \in P$ there is a $\bar{p} \in P_{\alpha}$ such that every $q \in P_{\alpha}$ stronger than $\bar{p}$ is compatible with $p$. Note that conditions $p$ and $q$ are compatible if and only if no element of $p-q$ is 
less than any element of $q$ and no element of $q-p$ is less than any element of $p$.

Let $p \in P$. Let $Z$ be the (finite) set $\left\{x \in S_{\alpha}: x \leq a\right.$ for some $\left.a \in p\right\}$, and let $u \in S_{\alpha}$ be a red point such that $u>x$ for all $x \in Z$ and $(x, u)$ is red for all $x \in p \cap S_{\alpha}$. Such a $u$ exists as $S_{\alpha}$ is an elementary submodel. Now let $\bar{p}=\left(p \cap S_{\alpha}\right) \cup\{u\}$.

Clearly, $\bar{p}$ is a condition in $P_{\alpha}$. Let $q \in P_{\alpha}$ be stronger than $\bar{p}$, and let us show that $q$ and $p$ are compatible. First, let $x \in q-p$ and $y \in p$. We claim that $x$ is not less than $y$ : since $q$ is stronger than $\bar{p}, x$ is not less than $u$, hence $x \notin Z$ and because $x \in S_{\alpha}$, the claim follows.

Second, let $x \in q$ and $y \in p-q$. We claim that $y$ is not less than $x$ : this is because $x \in S_{\alpha}, y \notin S_{\alpha}$, and since $S_{\alpha}$ is an elementary submodel and $x$ has finitely many predecessors, all $z<x$ are in $S_{\alpha}$.

Hence $p$ and $q$ are compatible.

We shall now outline how the above proof is modified to show that (3) implies (2) in the Cohen model. For instance, let $k=2$ and $r=3$. The above proof produces, in fact, a homogeneous cofinal set $H$ such that $D \cup\{H\}$ has the finite intersection property. (For every condition and every $A \in D$ there exists a stronger condition $q$ such that $q \cap A \neq \varnothing$.)

Let $F$ be a partition of $[S]^{3}$ into \{red, green $\}$. For each $a \in S$, let $F_{a}$ be the partition of $[S]^{2}$ given by $F_{a}(x, y)=F(a, x, y)$. Let $D$ be an ultrafilter on $S$ as before, and let $P_{a}$ denote the forcing that produces a homogeneous cofinal set for $F_{a}$. The product of $\left\{P_{a}: a \in S\right\}$ is isomorphic to adding $\aleph_{1}$ Cohen reals and if $\left\{H_{a}: a \in S\right\}$ are the generic homogeneous cofinal sets then $D \cup\left\{H_{a}: a \in S\right\}$ has the finite intersection property.

We may therefore assume that the sets $H_{a}$ are in the ground model, and $H_{a} \in D$ for each $a \in S$. We say that $a \in S$ is red, if $H_{a}$ is red-homogeneous; otherwise $a$ is green. Assuming that almost all $a \in S$ are red, a forcing condition is a finite red-homogeneous set of red points. This forcing produces a cofinal homogeneous set for the partition $F$, and is equivalent to adding $\aleph_{1}$ Cohen reals.

We shall now prove that (1) implies (3) in the Cohen model $V[G]$. So let $S \in V[G]$ be a directed partially ordered set of size $\aleph_{1}$ and assume that $S$ has the partition property. Consider the forcing notion $P$ that adds, with finite conditions, a generic partition of $[S]^{2}$ :

The forcing conditions in $P$ are functions whose domain is a finite subset of $[S]^{2}$, with values $\{$ red, green $\}$, and let $\dot{F}$ be the canonical name for a $P$-generic set. Clearly, $P$ is equivalent to adding $\aleph_{1}$ Cohen reals, and if $Q$ is the forcing that adds $\aleph_{2}$ Cohen reals, we have $Q \times P \simeq Q$. We shall prove:

Lemma. $P$ forces that if $\dot{F}$ has a cofinal homogeneous set, then $S$ has finite character.

Granted the lemma, we complete the proof of Theorem 2 as follows: Let $\dot{S}$ be a $Q$-name for $S \in V[G]$, and let $R$ be the forcing such that $V^{Q}=V[\dot{S}]^{R}$. 
We have $R \simeq Q$ and so $R \simeq R \times P$. The assumption is that $R$ (and therefore $R \times P$ ) forces that every partition of $S$ has a cofinal homogeneous set. Hence $R \times P$ forces that $\dot{F}$ has a cofinal homogeneous set, and it follows from the lemma that $R \times P$ forces that $S$ has finite character. Hence in $V[G], S$ has finite character.

Proof of Lemma. Let $\dot{H}$ be a $P$-name for a cofinal homogeneous set for $\dot{F}$, and assume that $P$ forces that $[\dot{H}]^{2}$ is green. Let $S_{\alpha}, \alpha<\omega_{1}$, be an elementary chain of countable submodels of $(S,<, P, \Vdash, \dot{F}, \dot{Q})$. First we claim that every condition forces the following: For every $\alpha$, if $a \in \dot{H}-S_{\alpha}$ then the set $\left\{x \in \dot{H} \cap S_{\alpha}: x<a\right\}$ is finite.

So let us assume otherwise, and let $a \notin S_{\alpha}$ and $p \in P$ be such that $p \Vdash$ $a \in \dot{H}$ and that $p \Vdash\left\{x \in \dot{H} \cap S_{\alpha}: x<a\right\}$ is infinite. There is therefore some $x<a, x \in S_{\alpha}$ such that $(x, a) \notin \operatorname{dom} p$ and that some $q$ stronger than $p$ forces $x \in \dot{H}$. Since $S_{\alpha}$ is an elementary submodel, there is some $q$ stronger than the restriction of $p$ to $\left[S_{\alpha}\right]^{2}$ such that $\operatorname{dom}(q) \subset\left[S_{\alpha}\right]^{2}$ and that $q$ forces $x \in \dot{H}$. Now $q$ and $p$ are compatible conditions, and moreover, $(x, a)$ is not in the domain of $q \cup p$, so let $r$ be the extension of $p \cup q$ that forces that $(x, a)$ is red. Then $r \Vdash(x \in \dot{H}$ and $a \in \dot{H}$ and $(x, a)$ is red $)$, which is a contradiction since $x<a$ and $[\dot{H}]^{2}$ is forced to be green.

Now we shall construct, in $V^{P}$, a cofinal subset $C$ of $H$ such that each $a \in C$ has only finitely many predecessors in $C$. For each $\alpha$, let $a_{\alpha 0} \in S_{\alpha+1}-S_{\alpha}$ be, if it exists, an element of $H$ that is not below any $x \in H \cap S_{\alpha}$. Then let $a_{\alpha n}, n<\omega$, be an increasing sequence starting with $a_{\alpha 0}$, cofinal in $H \cap S_{\alpha+1}$. Finally, let $C=\left\{a_{\alpha n}: \alpha<\omega_{1}, n<\omega\right\}$.

The set $C$ is cofinal in $H$. If $a_{\alpha n} \in C$, then by the claim proved above, $a_{\alpha n}$ has only finitely many predecessors in $C \cap S_{\alpha}$, and because $a_{\beta 0}$ is not less than $a_{\alpha n}$ for any $\beta>\alpha, a_{\alpha n}$ has only finitely many predecessors in $C$.

\section{Proof of LAVER's Theorem}

Let $a_{\alpha}$ and $\left(M_{\alpha}, H_{\alpha}\right), \alpha<\kappa$, enumerate, respectively, the set $[\kappa]^{<\omega}$ and the set of all pairs $(M, H)$ where $M \in[\kappa]^{\leq \aleph_{0}}$ and $H \subseteq[M]^{<\omega}$ is cofinal in $[M]^{<\omega}$. Furthermore, assume that $a_{\alpha} \subseteq \alpha$ and $M_{\alpha} \subseteq \alpha$ for all $\alpha$.

We construct a cofinal set $S=\left\{s_{\alpha}: \alpha<\kappa\right\}$ and a partition $F:[S]^{2} \rightarrow$ $\{1,2\}$ as follows: Let $\alpha<\kappa$. Let $b_{0}=a_{\alpha} \cup\{\alpha\} ; \alpha$ is the largest element of $b_{0}$. Choose, if possible, two distinct elements $c_{0}$ and $d_{0}$ of $H_{\alpha}$, and let $b_{1}=b_{0} \cup c_{0} \cup d_{0}$. Note that $\alpha$ is the largest element of $b_{1}$. Let $\alpha_{1}$ be the largest element of $b_{1}$ below $\alpha$, and choose, if possible, $c_{1}$ and $d_{1}$ in $H_{\alpha_{1}}$, distinct from $c_{0}$ and $d_{0}$ and from each other, and let $b_{2}=b_{1} \cup c_{1} \cup d_{1}$. Let $\alpha_{2}$ be largest in $b_{2}$ below $\alpha_{1}$, and choose $c_{2}, d_{2}$ in $H_{\alpha_{2}}$ distinct from $c_{0}, d_{0}$, $c_{1}, d_{1}$. This procedure terminates after finitely many, say $k$, steps, and we let $s_{\alpha}=b_{k}$. 
For each $i \leq k$, let $F\left(c_{i}, s_{\alpha}\right)=1$ and $F\left(d_{i}, s_{\alpha}\right)=2$, provided $c_{i}$ and $d_{i}$ are defined. Note that $\max s_{\alpha}=\alpha$, and that if $\beta$ is the $i$ th largest element of $s_{\alpha}$ and if $M_{\beta}$ is infinite then $c_{i}$ and $d_{i}$ are defined; hence there exist $c$ and $d$ in $H_{\beta}$ such that $F\left(c, s_{\alpha}\right)=1$ and $F\left(d, s_{\alpha}\right)=2$.

Let $S=\left\{s_{\alpha}: \alpha<\kappa\right\}$, and let $F$ be a partition of $[S]^{2}$ that satisfies the conditions specified above. We claim that no cofinal subset of $S$ is homogeneous for $F$.

Thus let $H$ be a cofinal subset of $S$. There exists an infinite countable set $M \subset \kappa$ such that $H \cap[M]^{<\omega}$ is cofinal in $[M]^{<\omega}$; let $\beta<\kappa$ be such that $M_{\beta}=M$ and $H_{\beta}=H \cap[M]^{<\omega}$. As $H$ is cofinal, there is an $x \in H$ such that $\beta \in x$; as $H \subseteq S$, there is some $\alpha$ such that $x=s_{\alpha}$.

Since $M_{\beta}$ is infinite, there exist $c, d \in H_{\beta}$ such that $F\left(c, s_{\alpha}\right)=1$ and $F\left(d, s_{\alpha}\right)=2$. Hence $H$ is not homogeneous for $F$.

\section{OPEN PROBLEMS}

(1) $\left[\aleph_{2}\right]^{<\omega} \rightarrow(\text { cofinal subset })_{2}^{2}$ (in ZFC),

(2) $\left[\aleph_{1}\right]^{<\omega} \rightarrow(\text { cofinal subset })_{2}^{3}$ (in ZFC),

(3) Is it consistent that there exists a directed partial ordering of size $\aleph_{1}$ that does not have finite character but has the partition property?

(4) $[A]^{<\omega} \rightarrow(\text { cofinal subset })_{k}^{r}$,

for all infinite sets $A$ and all integers $r, k \geq 2$.

\section{REFERENCES}

1. F. Galvin, seminar notes from U.C.L.A.

2. R. Graham, B. Rothschild, and J. Spencer, Ramsey Theory, Wiley, New York, 1980.

3. T. Jech, Some combinatorial problems concerning uncountable cardinals, Ann. Math. Logic 5 (1973), 165-198.

4. R. Laver, private communication.

ABSTRACT. Every partition of $\left[\left[\omega_{1}\right]^{<\omega}\right]^{2}$ into finitely many pieces has a cofinal homogeneous set. Furthermore, it is consistent that every directed partially ordered set satisfies the partition property if and only if it has finite character.

Department of Mathematics, The Pennsylvania State University, University Park, Pennsylvania 16802 and Mathematical Sciences Research Institute, Berkeley, CaliforNIA 94720

Department of Mathematics, The Hebrew University, Jerusalem, IsRael and Mathematical Sciences Research Institute, Berkeley, California 94720 